\title{
Um mapeamento de teses e dissertações sobre o processo de avaliação de Objetos de Aprendizagem: uma análise de conteúdo
}

Rosiney Rocha Almeida

Carlos Fernando Araújo Jr

Andréa Carla Leite Chaves

Cíntia Aparecida Bento dos Santos

\begin{abstract}
Resumo
Este artigo apresenta um mapeamento e evidencia a abordagem da avaliação pedagógica de Objetos de Aprendizagem (OA) em teses e dissertações depositadas no banco de teses da Capes no período de 2002 a 2011. A metodologia utilizada na pesquisa documental dessas publicações foi a Análise de Conteúdo de Bardin (2009).A análise da categoria, que trata dos objetivos das pesquisas, apontou para o fato de que o processo de avaliação de OA tem sido tratado como parte final do processo de desenvolvimento dos OA. São poucas as pesquisas que se ocupam exclusivamente da avaliação dos objetos, isso talvez seja, em parte, devido ao uso relativamente recente desses recursos digitais no contexto da sala de aula.Observou-se ainda que não há padronização dessas avaliações ou uma tendência específica, dada a diversidade das fundamentações teóricas e dos métodos utilizados para estes fins.

Palavras-chave: avaliação pedagógica de objetos de aprendizagem, análise de conteúdo, objetos de aprendizagem.
\end{abstract}

\begin{abstract}
A mapping of theses and dissertations on the evaluation process of Learning Objects: a content analysis

This article presents a mapping and highlights the approach of pedagogical evaluation of Learning Objects in theses and dissertations deposited at the Bank of theses of Capes in the period from 2002 to 2011. The methodology used in documentary research of these publications was the Analysis of Content of Bardin (2009). The analysis of category, which deals with the objectives of the research, pointed to the fact that the assessment process of Learning Objects has been treated as the final part of the process of development of Learning Objects. There is little research dealing exclusively with evaluation of objects, that may be partly due to the relatively recent use of digital resources in the context of the classroom. It was observed that there is no standardization of these assessments or a particular trend, given the diversity of theoretical foundations and of the methods used for these purposes.
\end{abstract}

Keywords: pedagogical evaluation of learning objects, content analysis, learning objects. 


\section{Introdução}

Conforme Boff e Reategui (2005), Nascimento (2007), Souza et al (2007), existe a necessidade de contar, para elaboração de Objetos de Aprendizagem (OA), com uma equipe multidisciplinar que tenha profissionais que respondam, de forma coerente, pelo caráter tanto pedagógico, quanto técnico desses objetos. A necessidade de uma equipe multidisciplinar, nesse processo, se dá também por considerar que, conforme afirma Nascimento (2007), autores e equipes de produção muitas vezes se deixam influenciar pelo potencial lúdico, em detrimento do potencial de aprendizagem de seus produtos, resultando em atividades que entretêm o aluno, mas com as quais ele não aprende.

Vieira (1999) destaca também que não se concebe a ideia de avaliar um software educativo considerando somente a beleza gráfica, em que são criados ambientes graficamente sofisticados, mas que desconhecem a longa trajetória do aprendiz para construir seus conhecimentos. Dessa forma, justifica-se a necessidade de avaliar OA não apenas do ponto de vista técnico, mas também quanto à sua qualidade pedagógica.

A motivação para o presente estudo se deu pelo fato de que diversos autores, entre eles Boff e Reategui (2005), Souza et al (2007) e Romero, Andrade e Pietrocola (2009), vêm discutindo a necessidade de se ampliar o debate acerca da avaliação pedagógica de Objetos de Aprendizagem. De acordo com Krauss e Ally (2005), é fundamental assegurar que os educadores estejam utilizando recursos que sejam altamente avaliados e acrescentem valor ao processo de ensino.

Contudo, Brandão (2004) e Gama (2007) afirmam que existe uma carência de modelos e métodos para a avaliação da qualidade das informações e conteúdos presentes nos objetos disponibilizados na internet. Dessa forma, ter a certeza de que esses objetos utilizados para fins educacionais são eficazes e possuem quesitos básicos de qualidade para o processo de ensino e aprendizagem torna-se um dos maiores desafios para os professores que adotam esses recursos em sua prática docente.

A tarefa de selecionar aspectos a serem considerados na avaliação de um Objeto de Aprendizagem é complexa, tendo em vista a multiplicidade de fatores tanto educacionais, quanto técnicos envolvidos nesse processo. Torna-se importante, portanto, conhecer de que forma e em que condições esses recursos têm sido produzidos, avaliados e divulgados em dissertações e teses no cenário nacional.

Um caminho possível é a realização de um levantamento das pesquisas produzidas a esse respeito, o que possibilita a efetivação do balanço da pesquisa de uma determinada área, pois, pesquisas desse tipo podem indicar a compreensão do estado atingido pelo conhecimento a respeito de um dado tema - sua amplitude, tendências teóricas e vertentes metodológicas (ROMANOWSKI \&ENS, 2006, pag. 40). 
"Esses estudos são justificados por possibilitarem uma visão geral do que vem sendo produzido na área e uma ordenação que permite aos interessados perceberem a evolução das pesquisas na área, bem como suas características e foco, além de identificar as lacunas ainda existentes" (ROMANOWSKI \& ENS, 2006, p. 41).

Foram levantadas, para fim desta pesquisa, 05 (cinco) categorias de análise para o material coletado, listadas abaixo, descritas com detalhes nos procedimentos metodológicos:

1) Envolvidos no processo da avaliação;

2) origem (fonte) das pesquisas: programas ou departamentos que tratam dessa temática;

3) proposta das pesquisas;

4) áreas de atuação relacionadas à avaliação de $O A$;

5) distribuição geográfica das pesquisas.

Este estudo se propõe a realizar um mapeamento de pesquisas produzidas no cenário acadêmico que tenham como temática a avaliação pedagógica de OA. Para tanto, foram mapeadas teses e dissertações depositadas no banco de teses da Capes no período que compreende os anos de 2002 e 2011. Essa delimitação temporal se deve ao fato de que a utilização das tecnologias cresceu significativamente nos últimos dez anos. Assim, espera-se proporcionar uma visão geral do desenvolvimento dessa temática de pesquisa nos últimos 10 anos.

\section{Procedimentos teórico-metodológicos}

Para Willey (2001), Objeto de Aprendizagem (OA) é qualquer recurso digital que possa ser utilizado para dar suporte ao ensino. Gama \& Scheer (2005) definem como elemento de uma nova metodologia de ensino e aprendizagem baseada no uso do computador e da internet, fundamentados em uma linguagem clara, com possibilidade de usabilidade para diversos contextos.

Portanto, objeto de Aprendizagem trata-se de um recurso digital que pode auxiliar o processo de ensino e aprendizagem. Como exemplos desses recursos, podemos citar imagens, animações, simulações, vídeos, etc. Ao fazermos uso de uma simulação sobre a digestão, por exemplo, podemos tornar um processo, que é naturalmente complexo, mais próximo do "real" e"descomplicado" (ALMEIDA, 2010).

São inúmeras as situações em que os OA têm sido utilizados. Listamos como exemplo o seu uso no ensino de física quântica (SALES \& VASCONCELOS FILHO, 2008; BETZ et al, 2009); de bioquímica (VIEIRA, 2011) e de Ciências (SILVEIRA, 2012). Existe uma variedade de emprego 
desses recursos para o ensino de Matemática (VASCONCELOS, 2010; OLIVEIRA, 2011; SANTOS, 2011; OLIVEIRA, 2012; COELHO, 2012 e MONZON, 2012. Também existem registros da utilização de OA como recurso pedagógico em contextos inclusivos (BARDY et al, 2013), cujo objetivo é promover uma reflexão sobre a inclusão, contribuir para uma mudança na forma como os docentes percebem a diversidade, bem como para uma ressignificação da prática docente (BISOL \& VALENTINI, 2012).

A coleta e análise de resumos de teses e dissertações possibilitam apontar caminhos que vêm sendo tomados e as restrições sobre o campo em que se move a pesquisa; além de identificar experiências inovadoras e reconhecer as contribuições da pesquisa na constituição de propostas na área (Romanowski \& Ens, 2006). Ainda, segundo os autores, essa análise permite examinar as ênfases e temas abordados nas pesquisas; os referenciais teóricos que subsidiaram as investigações; a relação entre o pesquisador e a prática pedagógica; as sugestões e proposições apresentadas pelos pesquisadores e as contribuições da pesquisa para mudança e inovações da prática pedagógica.

O presente trabalho se constitui em uma pesquisa com cunho qualitativo em consonância com Bardin (2009), que se efetiva com base na análise da presença ou ausência de uma dada característica do conteúdo. Foram analisadas teses e dissertações depositadas no banco de teses da Capes no período que compreende 2002 e 2011. O acesso ao banco de teses e dissertações da CAPES ocorreu nos meses de setembro e outubro de 2012.

Para a análise dos resumos das teses e dissertações que tratam da avaliação pedagógica de OA, seja através da apresentação e discussão do processo ou de propostas de metodologias para esse fim, utilizou-se como metodologia a análise do conteúdo que, segundo Bardin (2009), trata-se de:

"um conjunto de técnicas de análise das comunicações visando obter, por procedimentos sistemáticos e objetivos de descrição do conteúdo das mensagens, indicadores (quantitativos ou não) que permitam a inferência de conhecimentos relativos às condições de produção/recepção (variáveis inferidas) destas mensagens" (BARDIN, 2009, p.44).

Quando necessário, fez-se a análise do texto completo, pois, ao concordar com Megid (1999), os resumos ampliam um pouco mais as informações disponíveis, porém, por serem muito sucintos e, em muitos casos, mal elaborados ou equivocados, não são suficientes para a divulgação dos resultados e das possíveis contribuições dessa produção para a melhoria do sistema educacional. Somente com a leitura completa ou parcial do texto final da tese ou dissertação é que esses aspectos podem ser percebidos. 
2.1 Pré-análise: Fez-se em primeiro lugar a preparação do material através da busca de resumos no banco de teses da Capes no período de 2002 a 2011, através de palavras-chave que possibilitaram chegar a um levantamento de 30 pesquisas. Após a coleta, realizou-se uma leitura flutuante em que, segundo Bardin (2009), é possível surgir, através dela, hipóteses ou questões norteadoras que permitem observar convergências e divergências entre o material analisado e o propósito da pesquisa.

A análise textual dos resumos foi feita através de fichamentos, que permitiram identificar os elementos fundamentais da pesquisa, tais como: título, autores, linha de pesquisa, ano, instituição de origem, foco temático, objetivos, referencial teórico, procedimentos metodológicos, resultados obtidos e contribuição para a área das pesquisas.

2.2 Hipótese: Através da análise de conteúdo pode-se confirmar ou não uma hipótese, quando se considera que hipóteses, sob a forma de questões ou de afirmações provisórias, servindo de diretrizes apelarão para o método de análises sistemáticas para serem verificadas no sentido de uma possível confirmação ou não. Assim, será feita a análise de conteúdo para servir de prova (BARDIN, 2009, p. 30) para a hipótese levantada nesta pesquisa: por ser bastante incipiente no que diz respeito à avaliação pedagógica de $O A$, o cenário nacional ainda não está consolidado, nem tampouco possui uma padronização desse processo.

2.3 Material selecionado: A partir da leitura, foi possível selecionar, e descartar pesquisas que, apesar de tratarem de avaliação de OA, não tratavam de sua avaliação pedagógica, propósito deste trabalho. Obteve-se um total de 09 (nove) trabalhos selecionados dos quais 02 (dois) são teses; e 07 (sete) dissertações, que constituem o corpus da pesquisa, conforme estão descritas no Quadro 01, a seguir:

Quadro 1- Teses/dissertações relacionadas à avaliação pedagógica de OA no banco da Capes no período de 2002 a 2011.

\begin{tabular}{|c|c|c|c|c|}
\hline & Título & Universidade & Classificação & Ano \\
\hline 1) & $\begin{array}{l}\text { Desenvolvimento e avaliação de } \\
\text { objeto virtual de aprendizagem } \\
\text { interativo sobre o raciocínio } \\
\text { diagnóstico em enfermagem } \\
\text { aplicado ao recém-nascido pré- } \\
\text { termo. }\end{array}$ & $\begin{array}{l}\text { UNIVERSIDADE SÃO } \\
\text { PAULO }\end{array}$ & Tese & 2010 \\
\hline 2) & $\begin{array}{l}\text { Avaliação de Objetos de } \\
\text { Aprendizagem: cruzando } \\
\text { caminhos e produzindo novos } \\
\text { olhares. }\end{array}$ & $\begin{array}{l}\text { UNIVERSIDADE FEDERAL } \\
\text { DE UBERLÂNDIA }\end{array}$ & Dissertação & 2009 \\
\hline
\end{tabular}




\begin{tabular}{|c|c|c|c|c|}
\hline 3) & $\begin{array}{l}\text { Avaliação Pedagógica de } \\
\text { Objetos de Aprendizagem do } \\
\text { Ensino de Geografia na Visão } \\
\text { dos Professores }\end{array}$ & $\begin{array}{l}\text { UNIVERSIDADE } \\
\text { ESTADUAL DO CEARÁ }\end{array}$ & Dissertação & 2010 \\
\hline 4) & $\begin{array}{l}\text { ENERGIA, ALIMENTAÇÃO E } \\
\text { DESEMPENHO NA ATIVIDADE } \\
\text { FíSICA: elaboração e avaliação } \\
\text { de um Objeto de Aprendizagem } \\
\text { multimodal para o ensino de } \\
\text { Bioquímica }\end{array}$ & $\begin{array}{l}\text { PONTIFÍCIA } \\
\text { UNIVERSIDADE CATÓLICA } \\
\text { DE MINAS GERAIS }\end{array}$ & Dissertação & 2011 \\
\hline 5) & $\begin{array}{l}\text { Avaliação de jogos voltados } \\
\text { para a disseminação do } \\
\text { conhecimento }\end{array}$ & $\begin{array}{l}\text { UNIVERSIDADE FEDERAL } \\
\text { DE SANTA CATARINA }\end{array}$ & Tese & 2011 \\
\hline 6) & $\begin{array}{l}\text { Avaliação de software } \\
\text { educacional para ensino- } \\
\text { aprendizagem de Inglês para a } \\
\text { escola pública: coletando } \\
\text { subsídios avaliativos }\end{array}$ & $\begin{array}{l}\text { PONTIFÍCIA } \\
\text { UNIVERSIDADE CATÓLICA } \\
\text { DE SÃO PAULO }\end{array}$ & Dissertação & 2007 \\
\hline 7) & $\begin{array}{l}\text { Avaliação de usabilidade em } \\
\text { softwares educativo. }\end{array}$ & $\begin{array}{l}\text { UNIVERSIDADE } \\
\text { ESTADUAL DO CEARÁ }\end{array}$ & Dissertação & 2010 \\
\hline 8) & $\begin{array}{l}\text { Desenvolvimento e análise da } \\
\text { utilização de um sistema } \\
\text { interativo para a aprendizagem } \\
\text { de Genética }\end{array}$ & $\begin{array}{l}\text { UNIVERSIDADE FEDERAL } \\
\text { DO RIO DE JANEIRO }\end{array}$ & Dissertação & 2003 \\
\hline 9) & $\begin{array}{l}\text { Jogos eletrônicos educativos: } \\
\text { instrumento de avaliação } \\
\text { focado nas fases iniciais do } \\
\text { processo de design. }\end{array}$ & $\begin{array}{l}\text { UNIVERSIDADE FEDERAL } \\
\text { DO PARANÁ }\end{array}$ & Dissertação & 2010 \\
\hline
\end{tabular}

(Fonte: Elaborado pela pesquisadora, 2012.)

Obedeceu-se às regras de exaustividade (BARDIN, 2009) ao se fazer a busca pelo maior número de palavras-chave possíveis; de representatividade, visto que, apesar de se tratar de uma amostra pequena, os documentos incluídos na amostra são representativos para a pesquisa e pertinentes aos objetivos da análise, podendo cobrir o campo de pesquisa acerca da avaliação de $\mathrm{OA}$, esses foram buscados no banco de teses e dissertações da capes, em que são 
disponibilizados resumos de teses e dissertações apresentadas nos programas de Pós-Graduação do país em um período de 10 anos; de homogeneidade, todas as teses/dissertações coletadas referem se ao mesmo tema, e foram obtidas pelos mesmos critérios de busca e pelo mesmo pesquisador; e enfim, de pertinência: em que os objetivos da análise são adaptados ao conteúdo e objetivo da pesquisa.

2.4 Categorias para análise: Fez-se uma releitura cuidadosa dos trabalhos aqui investigados com a finalidade de definir a unidade de análise (elemento unitário de conteúdo a ser submetido posteriormente à classificação), para posterior levantamento das categorias a serem analisadas. De tal modo foram definidas as seguintes unidades de análise:

1) Contexto das avaliações;

2) Enfoque das avaliações;

Dentro do critério CONTEXTO das avaliações pode se estabelecer as seguintes categorias:

A) Origem (fonte) das pesquisas: esta categoria permitiu analisar quais são os programas ou os departamentos que têm tratado dessa temática.

B) Envolvimento no processo de avaliação: buscou-se através dessa categoria analisar quem são os envolvidos no processo de avaliação pedagógica de $O A$, se somente alunos, somente professores, professores e alunos, ou outros profissionais.

Dentro do critério ENFOQUE das avaliações, estabeleceram-se as seguintes categorias:

A) Proposta das pesquisas: a partir dessa categoria, buscou-se entender se as pesquisas que tratam da avaliação de OA trazem apenas o processo de avaliação como proposta de investigação ou se vem sendo tratado como parte de uma proposta maior de investigação;

B) Áreas de atuação relacionadas à avaliação de $O A$; que possibilitou analisar quais as áreas de atuação têm se dedicado a essa proposta.

C) Distribuição geográfica - que permitiu identificar a disposição dessas pesquisas nas regiões do país.

\section{Resultados}

Apresentamos alguns resultados a que chegamos nesta pesquisa em consonância com as proposições de Bardin (2009). De acordo com o autor, o gênero de resultados obtidos pelas técnicas de análise de conteúdo não pode ser tomado como prova irrefutável, mas constitui, apesar de tudo, uma ilustração que permite corroborar, pelo menos parcialmente, os pressupostos em causa. 
Foram encontradas, no período delimitado para este estudo, apenas 09 (nove) pesquisas que tratam especificamente da avaliação pedagógica de $\mathrm{OA}$, das quais, 02 (duas) são teses; e 07 (sete), dissertações.

Com relação aos programas ou departamentos em que são produzidas e tratadas as pesquisas relacionadas à avaliação pedagógica de OA observou-se que apenas 33,3\% são relacionados à educação ou ao ensino (Figura 01). Além disso, pode-se verificar também, que das duas pesquisas analisadas que têm como proposta a elaboração de metodologias para a avaliação desses recursos, apenas uma delas tem um profissional com formação pedagógica compondo a equipe, com o propósito de auxiliar nesse processo.

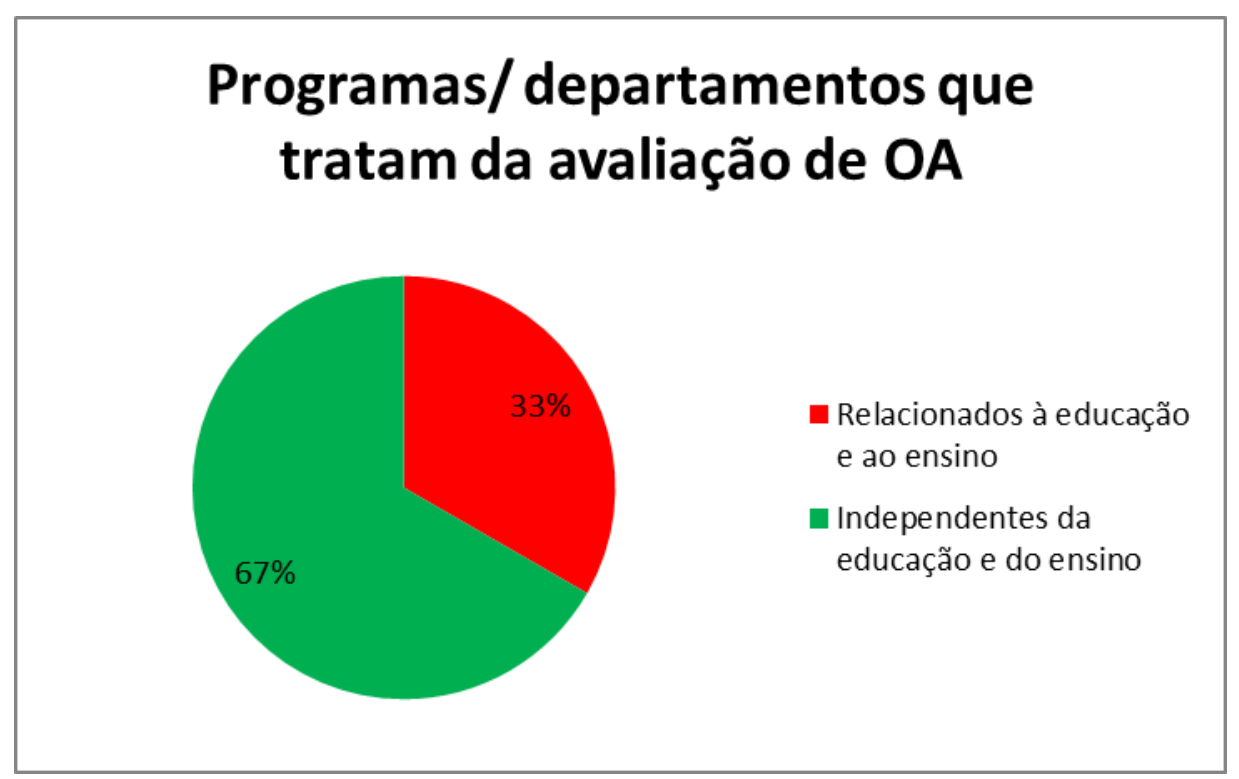

Figura 01: Programas/Departamentos que tratam da avaliação pedagógica de OA.

(Fonte: Dados da pesquisa, 2012.)

Com relação à análise dos sujeitos envolvidos no processo, conforme Figura 02 (dois), abaixo, é possível notar que, em alguns casos, a avaliação pedagógica de OA não fica a cargo do profissional competente para tal, isso pode comprometer, sobremaneira, a qualidade e eficiência desse recurso no processo de ensino-aprendizagem. Essa observação está de acordo com Silva (1997) ao admitir que esses programas têm mais em comum com a lógica do entretenimento do que com as necessidades pedagógicas e operacionais dos processos de aprendizagem, prevalecendo a lógica do programador e não a do aluno. Esses resultados apontam para um dos entraves atuais no que diz respeito ao desenvolvimento e avaliação de $O A$, uma vez que, conforme se observa no gráfico da figura 02 , esses objetos são normalmente pensados e avaliados por profissionais que, muitas vezes, desconhecem a trajetória do aluno no seu processo de aprendizagem. 


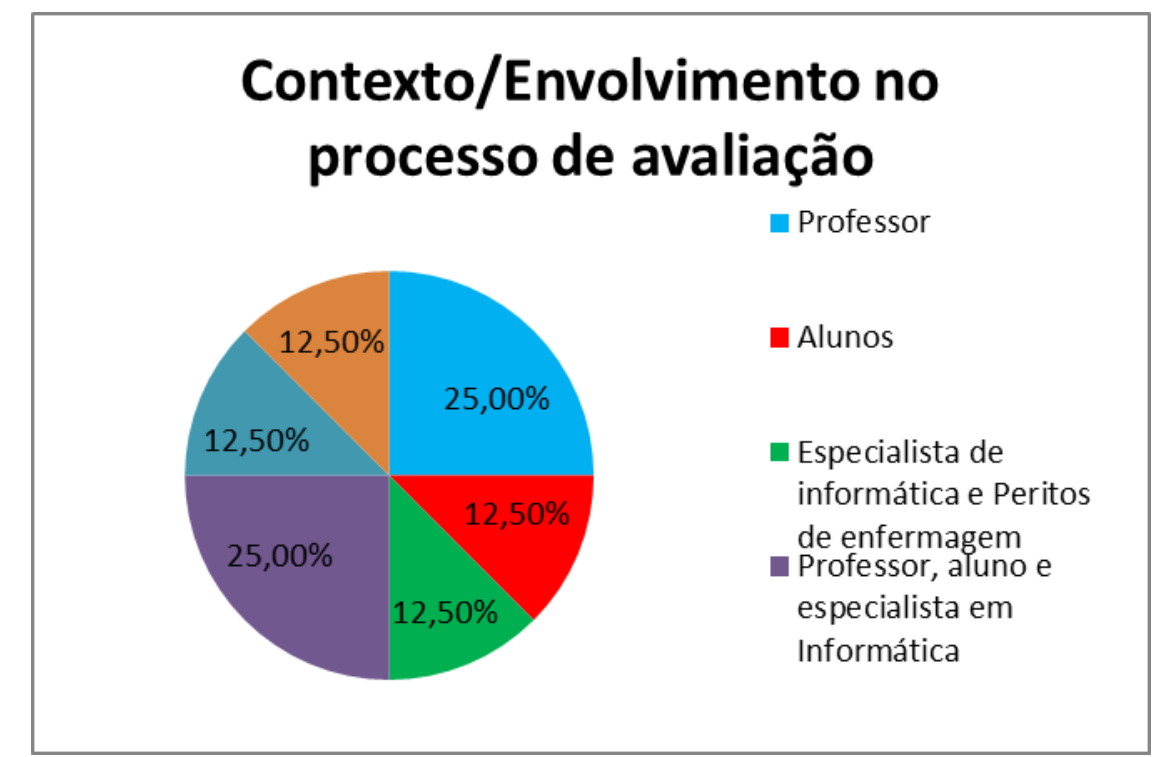

Figura 02: Profissionais envolvidos no processo de avaliação pedagógica de OA

Fonte: Dados da pesquisa, 2012

$\mathrm{Na}$ análise dos objetivos das pesquisas selecionadas para este estudo, apresentada na Figura 03 (três), notou-se que 33 \% se propuseram a desenvolver e avaliar OA; aproximadamente $45 \%$ trataram apenas da avaliação de AO; e $22 \%$ se detiveram na elaboração de propostas de metodologias ou instrumentos. Observou-se então que cerca de $80 \%$ se limitaram à avaliação em si (apresentação do processo/ método avaliativo/ resultados/ de avaliação de $O A$ ), enquanto pouco mais de $20 \%$ das pesquisas concentrou-se na proposta e validação de método e/ou instrumento de avaliação de OA.

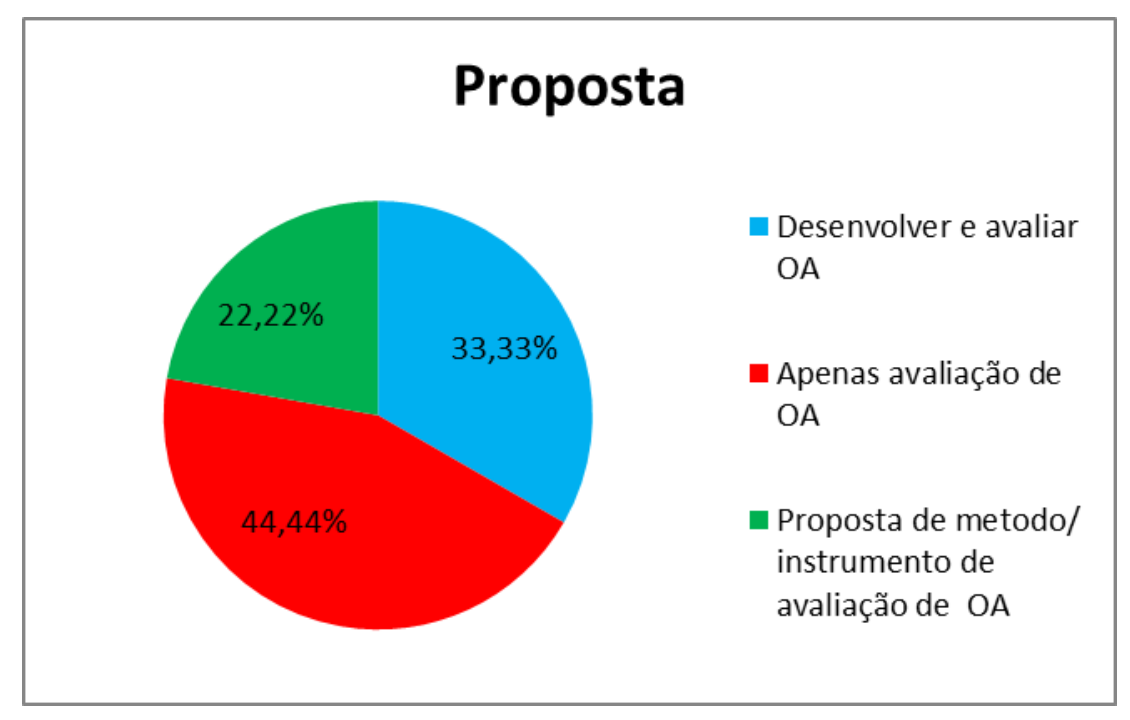

Figura 03: Proposta em relação à avaliação pedagógica de OA.

(Fonte: Dados da pesquisa, 2012.) 
No que se refere à fundamentação teórica e aos métodos utilizados nessas pesquisas, constatou-se que são bastante diversificados, fato que pode ser justificado pela diversidade dos programas que tratam do assunto, em que cada um adota sua vertente teórica e metodológica. Dada essa enorme diversidade, torna-se difícil prever os rumos desse processo no cenário nacional. Essa análise permite perceber que não existe uma tendência única para as pesquisas relacionadas à avaliação de $\mathrm{OA}$, e que essas são, ainda, muito diversificadas.

Foi feita também uma análise a respeito da área de atuação dos profissionais que participam da avaliação pedagógica dos $\mathrm{OA}$ e, conforme exposto na Figura 04, percebe-se que não existe uma predominância de uma área em detrimento de outra, o que reforça o caráter bastante heterogêneo dessas pesquisas atualmente no país.

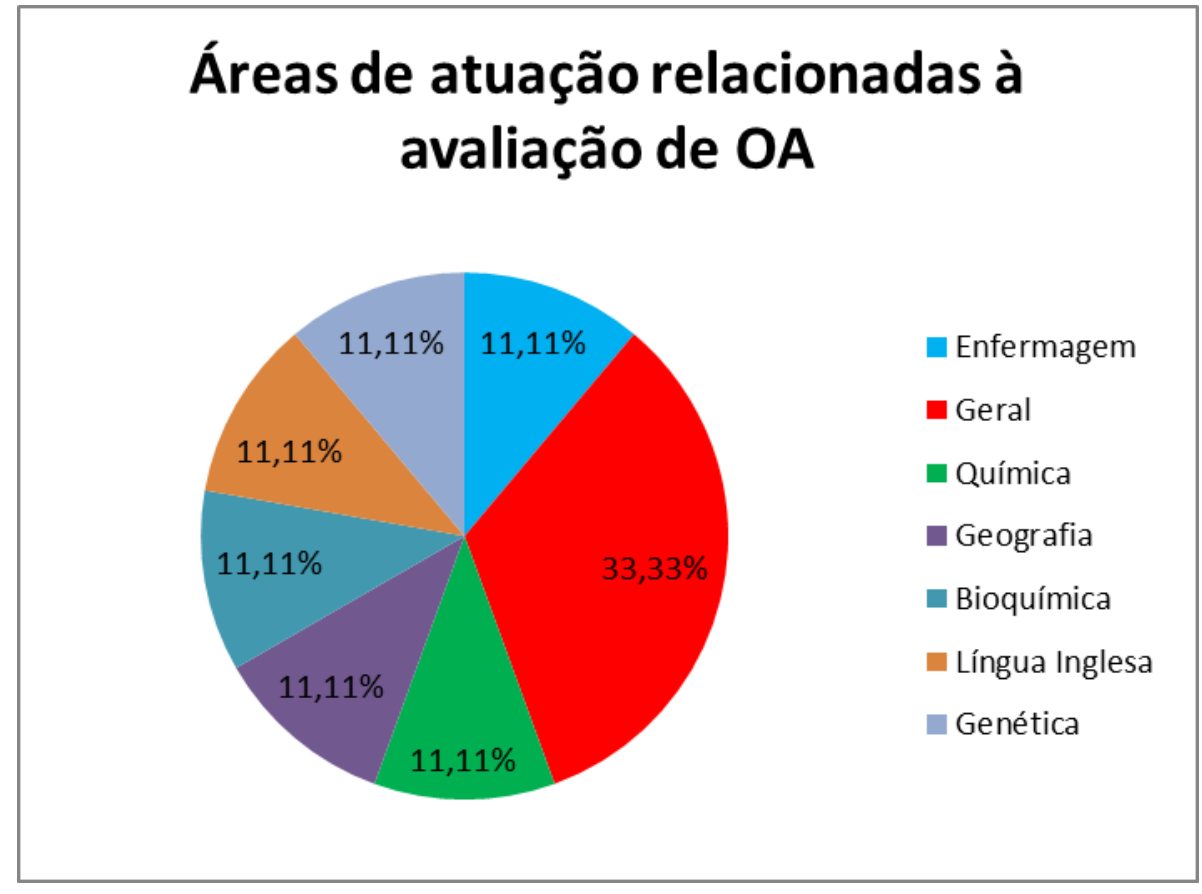

Figura 04: Áreas de atuação dos profissionais que participam da avaliação pedagógica De OA.

(Fonte: Dados da pesquisa, 2012.)

A análise da distribuição (Figura 05) permite constatar que existe uma predominância dessas pesquisas na Região Sudeste (aproximadamente 65\%). Pode-se observar também que não foi identificada nenhuma ocorrência nas regiões Centro-Oeste e Norte, o que pode ser explicado por uma menor ocorrência de universidades nessas regiões quando comparadas às demais. 


\section{Distribuição geográfica do local de realização do trabalho}

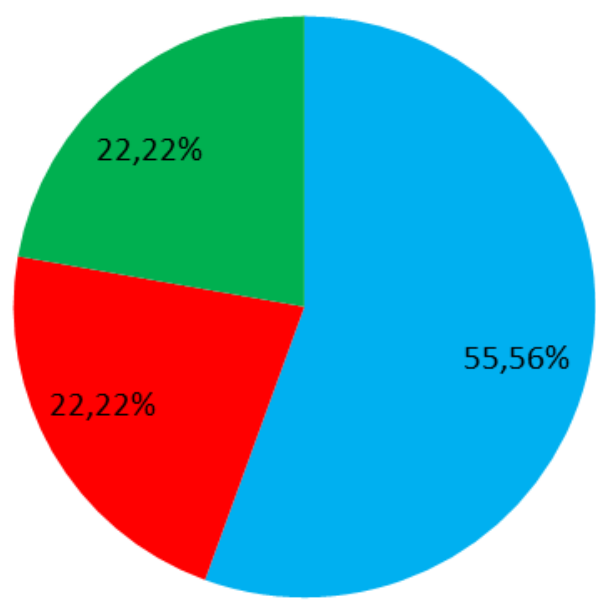

Figura 05: Distribuição geográfica do local de realização das pesquisas de avaliaçãopedagógica de $O A$.

(Fonte: Dados da pesquisa, 2012)

\section{Considerações gerais}

A análise de conteúdo das teses e dissertações investigadas nesta pesquisa corroborou a percepção de que existe uma carência no cenário nacional de trabalhos que tratem especificamente da avaliação pedagógica de $\mathrm{OA}$, seja no processo em si ou na proposta de metodologias para esse fim.

A ausência de uma avaliação que contemple os aspectos pedagógicos presentes nesses objetos ou a delegação dessa responsabilidade a profissionais sem a formação específica para esse fim podem comprometer a qualidade desses recursos. Muitas vezes, essa tarefa é atribuída a profissionais que desconhecem o processo cognitivo humano, intensificando o distanciamento entre a proposta e a real contribuição desses artefatos no processo de ensino-aprendizagem.

Observou-se uma heterogeneidade de vertentes teórico-metodológicas e diversidade de programas ou departamentos que tratam da avaliação pedagógica de OA. Isso aponta para a necessidade de se investir em novas pesquisas no sentido de contribuir para a padronização e aprimoramento do processo de avaliação desses objetos.

A análise da categoria que trata dos objetivos das pesquisas apontou para o fato de que o processo de avaliação de $O A$ tem sido tratado como parte final do processo de desenvolvimento 
dos OA. São poucas as pesquisas que se ocupam exclusivamente da avaliação dos objetos, o que pode estar relacionado ao uso recente desses recursos digitais no contexto da sala de aula.

\section{Referências}

ALMEIDA, R. R. Elaboração de um catálogo de objetos de aprendizagem digitais para o ensino do sistema digestório com ênfase no seu potencial como ferramenta de ensino e aprendizagem. 2010. 86 f. Dissertação (Mestrado em Ensino de Ciências e Matemática) - Pontifícia Universidade Católica de Minas Gerais, Belo Horizonte, 2010.

BARDIN, L. Análise de conteúdo. Lisboa: Edições 70, 2009.

BARDY, L. R.; HAYASHI, M. C. P. I.; SCHLLÜNZEN, E. T. M.; SEABRA JÚNIOR, M. O. Objetos de Aprendizagem como recurso pedagógico em contextos inclusivos: subsídios para a formação de professores a distância. Revista Brasileira de Educação Especial, v. 19, p. 273-288, 2013.

BETZ, M.;LIMA, I.; MUSSATTO, G. Dualidade onda-partícula: um objeto de aprendizagem baseado no interferômetro de Mach-Zehnder. Revista Brasileira de Ensino de Física, v. 31, n. 3, 2009.

BISOL, C. A.; VALENTINI, C. B. Desafios da inclusão: uma proposta para a qualificação de docentes no Ensino Superior via tecnologias digitais. Revista Portuguesa de Educação, v.25, n.2, p. 263-280, 2012.

BOFF, E.; REATEGUI, E. A importância do processo de avaliação de software educativo. In:SEMINÁRIO NACIONAL DE TECNOLOGIA NA EDUCAÇÃO, 2., 2005. Caxias do Sul. Anais ... Caxias do Sul, 2005. Disponível em: <http://ccet.ucs.br/dein/nase/snte2005.PDF> Acesso em: 10 dez. 2012.

BRANDÃO, E. J. R. Repensando modelos de avaliação de software educacional. 2004. [S.I.]. Disponível em: <http://www.minerva.uevora.pt/simposio/comunicacoes/artigo.html> Acesso em: 10 dez. 2012.

COELHO, V. L. C. D. Um objeto de aprendizagem para o ensino do conceito de limite de uma função. 2012.210 f. Dissertação (Mestrado em Modelagem Computacional e Tecnologia Industrial) - Faculdade de Tecnologia SENAI CIMATEC, Salvador, 2012.

GAMA, C. L. G. Método de construção de objetos de aprendizagem com aplicação em métodos numéricos. 2012. 196 f. Tese (Doutorado em Métodos Numéricos em Engenharia ) - Universidade Federal do Paraná, Curitiba, 2007. Disponível em:

<http://www.ppgmne.ufpr.br/arquivos/teses/9.pdf> Acesso em: 10 dez.2012.

GAMA, C. L. G.; SCHEER, S. Avaliação de objetos educacionais para educação a distância de engenharia: construção, reuso e avaliação. In: CONGRESSO INTERNACIONAL DE EDUCAÇÃO À DISTÂNCIA, 12, 2005, Florianópolis. Programação do 12 CIED... Florianópolis: ABED e UFSC, 
2005. v.1. p. 1-8. Disponível em: http://www.abed.org.br/congresso2005/por/pdf/159tcc3.pdf. Acesso em: 15 jan. 2013.

KRAUSS, F.; ALLY, M. A Study of the design and evaluation of a learning object and implications for content development: interdisciplinary. Journal of Knowledge and Learning Objects. California, v.1, p. 1-22, 2005.Disponívelem: <http://ijklo.org/Volume1/v1p001-022Krauss.pdf>. Acesso em: 5 dez. 2012.

MEGID NETO, J. Tendências da pesquisa acadêmica sobre o ensino de ciências no nível fundamental. 1999.114 f. Tese (Doutorado em Educação ) - Faculdade de Educação, Universidade Estadual de Campinas, Campinas, 1999.

MONZON, L. W. Números complexos e funções de variável complexa no ensino médio uma proposta didática com uso de objeto de aprendizagem. 2012. 134 f. Dissertação (Mestrado em Ensino de Matemática). Universidade Federal do Rio Grande do Sul, Porto Alegre, 2012.

NASCIMENTO, A. C. A. A. Objetos de aprendizagem: entre a promessa e a realidade. In: PRATA, C. L.; NASCIMENTO, A. C. A. A. (Org.). Objetos de aprendizagem: uma proposta de recurso pedagógico. Brasília: MEC/SEED, 2007. p. 135-146.

OLIVEIRA, A. L. Objeto de aprendizagem para desenvolvimento de habilidades de visualização e representação de secções cônicas: atividades para o ensino médio. 2011. 106 f. Dissertação (Mestrado em Ensino de Ciências e Matemática) - Pontifícia Universidade Católica de Minas Gerais. Belo Horizonte, 2011.

OLIVEIRA, I. P. S. Objeto de aprendizagem a luz dos pressupostos Vigotskianos para o ensinoaprendizagem de funções quadráticas no Ensino Médio. 2012. 124 f. Dissertação (Mestrado em Modelagem Computacional e Tecnologia Industrial) - Faculdade de Tecnologia SENAI CIMATEC, Salvador, 2012.

ROMANOWSKI,J.P.; ENS, R. T. As pesquisas denominadas do tipo "estado da arte" em educação. Revista Diálogo Educacional, PUCPR, v.6, p.37-50, 2006.

ROMERO, T. R. L.; ANDRADE, R. de; PIETROCOLA, M. Parâmetros para análise de roteiros de objetos de aprendizagem. In: SIMPÓSIO NACIONAL DE ENSINO DE FÍSICA, 18., 2009, Vitória. Resumos... Vitória, 2009. Disponível em: <http://www.sbf1.sbfisica.org.br/eventos/snef/xviii/sys/resumos/T0238-1.pdf>.

SALES, G. L.; VASCONCELOS, F. H. L.; CASTRO FILHO, J. A.; PEQUENO, M. C. Atividades de modelagem exploratória aplicada ao ensino de física moderna com a utilização do objeto de aprendizagem pato quântico. Revista Brasileira de Ensino de Física, São Paulo, v. 30, n.3, 2008.

SANTOS, J. J. A. Desenvolvimento de um objeto de aprendizagem para o ensino de conceitos de probabilidade. 2011. 76 f. Dissertação (Mestrado em Ensino de Ciências e Matemática) Universidade Estadual da Paraíba, João Pessoa, 2011. 
SILVA, D.; MARCHELLI, P. S. Informática e ensino: visão crítica do softwares educativos e discussão sobre as bases adequadas para o seu desenvolvimento. In: SIMPÓSIO NACIONAL DE ENSINO DE FíSICA, 12., 1997. Belo Horizonte. Atas... Belo Horizonte, v. 1, p. 517-525. Disponível em: <http://www.ufmt.br/ ufmtvirtual/textos/se_visao.htm>. Acesso: 21 dez. 2012.

SILVEIRA, F. S. A utilização de um objeto de aprendizagem sobre matriz elétrica para o ensino de Ciências. 2012. 102 f. Dissertação (Mestrado em Educação em Ciências Química da Vida e Saúde) - Universidade Federal do Rio Grande do Sul, Porto Alegre, 2012.

SOUZA, M. F. C et al. LOCPN: Redes de Petri Coloridas na Produção de Objetos de Aprendizagem. Revista Brasileira de Informática na Educação. Porto Alegre, v. 15, n. 3, p. 39-42. 2007. Disponível em: <http://www.br-ie.org/pub/index. php/rbie/article/view/16>. Acesso: 10 dez. 2012.

VASCONCELOS, M. H. S.; TOGNI, A. C. Aprendendo estatística no ensino médio e no Curso Técnico Agrícola em Agropecuária utilizando o objeto de aprendizagem EstatísticaNet. Circuito da Tecnologia da Informação do Instituto Federal Fluminense, v. 6, 2010.

VIEIRA, F. M. S. Avaliação de software educativo: reflexões para uma análise criteriosa. Campinas: EDUTECNET, 1999. Disponível em <http://www.edutec.net/Textos/Alia/MISC/edmagali2.htm>. Acesso em: 10 dez. 2012.

VIEIRA, M. M. S. Feira dos pesos: análise de um objeto de aprendizagem para o desenvolvimento do pensamento algébrico. 2011. 97 f. Dissertação (Mestrado em Computação Aplicada) Universidade Estadual do Ceará, Fortaleza, 2011.

WILEY, D.A. Connecting learning objects to instructional design theory: a definition a metaphor, and a taxonomy. 2001. Disponível em: http://reusability.org/read/chapters/wiley.doc. Acesso em: 15 jan. 2013.

Agradecemos a CAPES pelo apoio recebido.

Rosiney Rocha Almeida - Doutoranda em Ensino de Ciências e Matemática pela Pontifícia Universidade Cruzeiro do Sul. Professora do Instituto Federal Norte de Minas Gerais (IFNMG). Bolsista da Coordenação de Aperfeiçoamento de Pessoal de Nível Superior ( CAPES). Email: rrosyy@yahoo.com.br

Andréa Carla Leite Chaves - Pós-doutora no Centro de Pesquisas René Rachou-FIOCRUZ-MG. Professora adjunta e pesquisadora em Curso de Graduação e Pós-Graduação da Pontifícia Universidade Católica de Minas Gerais. Email: andreacarlachaves@yahoo.com.br 
Carlos Fernando Araujo Jr - Doutor em Física Teórica pela Universidade Estadual Paulista Júlio de Mesquita Filho (UNESP). Professor Pesquisador do Programa de Pós-graduação em Ensino de Ciências e Matemática pela Universidade Cruzeiro do Sul. Email: carlos.araujo@cruzeirodosul.edu.br

Cintia Aparecida Bento dos Santos - Doutora em Ensino de Ciências e Matemática pela Universidade Cruzeiro do Sul. Professora do Programa de Pós-Graduação em Ensino de Ciências e Matemática. Email: cintia.santos@cruzeirodosul.edu.br 